\title{
Supervised Exercise Immediately After Bariatric Surgery: the Study Protocol of the EFIBAR Randomized Controlled Trial
}

\author{
Enrique G. Artero ${ }^{1}$ (D) - Manuel Ferrez-Márquez ${ }^{2,3} \cdot$ María José Torrente-Sánchez $^{3}$ - Elena Martínez-Rosales ${ }^{1}$. \\ Alejandro Carretero-Ruiz ${ }^{1}$ - Alba Hernández-Martínez ${ }^{1}$ • Laura López-Sánchez ${ }^{1}$. Alba Esteban-Simón ${ }^{1}$. \\ Andrea Romero del Rey ${ }^{4}$ Manuel Alcaraz-lbáñez ${ }^{5}$. Manuel A. Rodríguez-Pérez ${ }^{1}$ • Emilio Villa-González ${ }^{6}$. \\ Yaira Barranco-Ruiz ${ }^{6}$. Sonia Martínez-Forte ${ }^{7}$. Carlos Castillo $^{8}$. Carlos Gómez Navarro ${ }^{9}$ - Jesús Aceituno Cubero ${ }^{9}$. \\ Raúl Reyes Parrilla ${ }^{9}$ José A. Aparicio Gómez ${ }^{9}$. Pedro Femia ${ }^{10}$ • Ana M. Fernández-Alonso ${ }^{7}$. \\ Alberto Soriano-Maldonado ${ }^{1}$
}

Received: 4 May 2021 /Revised: 25 June 2021 / Accepted: 1 July 2021

(C) The Author(s) 2021, revised 2021

\begin{abstract}
Background Previous studies have investigated weight loss caused by exercise following bariatric surgery. However, in most cases, the training program is poorly reported; the exercise type, volume, and intensity are briefly mentioned; and the sample size, selection criteria, and follow-up time vary greatly across studies.

Purpose The EFIBAR study aims to investigate over 1 year the effects of a 16-week supervised exercise program, initiated immediately after bariatric surgery, on weight loss (primary outcome), body composition, cardiometabolic risk, physical fitness, and quality of life in patients with severe/extreme obesity.

Material and Methods The EFIBAR study is a parallel-group, superiority, randomized controlled trial (RCT), comprising 80 surgery patients. Half of the participants, randomly selected, perform a 16-week supervised exercise program, including both strength and aerobic training, starting immediately after the surgery (7-14 days). For each participant, all primary and secondary outcomes are measured at three different time points: (i) before the surgery, (ii) after the intervention ( $\approx 4$ months), and (iii) 1 year after the surgery.
\end{abstract}

Key Points

- Exercise programs following bariatric surgery are, in most cases, poorly reported.

- EFIBAR study includes 80 patients and a 16-week supervised exercise program, starting 7-14 days after the surgery.

- Multiple health-related outcomes are assessed before surgery, at 4 months, and 1 year.

- This RCT will contribute to understand the effectiveness of exercise as a therapeutic complement to bariatric surgery.

Enrique G. Artero

artero@ual.es

1 Department of Education, Faculty of Education Sciences; SPORT Research Group (CTS-1024), CERNEP Research Centre, University of Almería, Almería, Spain

2 General and Bariatric Surgery Unit, Torrecárdenas University Hospital, Almería, Spain

3 Hospital Mediterráneo, Almería, Spain

4 Pediatric Unit, Torrecárdenas University Hospital, Almería, Spain

5 Department of Education and Health Research Centre, University of Almería, Almería, Spain
6 PROFITH Promoting Fitness and Health through Physical Activity Research Group, Department of Physical and Sports Education, Faculty of Education and Sport Sciences, Sport and Health University Research Institute (iMUDS), University of Granada, Melilla, Spain

7 Obstetrics and Gynecology Unit, Torrecárdenas University Hospital, Almería, Spain

8 Department of Economics and Business, SPORT Research Group (CTS-1024), CERNEP Research Centre, University of Almería, Almería, Spain

9 Unit of Cardiology, Torrecárdenas University Hospital, Almería, Spain

10 Department of Statistics and Operations Research, Faculty of Medicine, University of Granada, Granada, Spain 
Conclusion The EFIBAR study will provide new insights into the multidimensional benefits of exercise in adults with severe/ extreme obesity following bariatric surgery.

Trial Registration EFIBAR randomized controlled trial was prospectively registered at Clinicaltrials.gov (NCT03497546) on April 13, 2018.

Keywords Obesity $\cdot$ Bariatric surgery $\cdot$ Exercise $\cdot$ Randomized controlled trial $(\mathrm{RCT}) \cdot$ Protocol

\section{Introduction}

Bariatric surgery is an effective treatment option for reducing weight in people with extreme obesity [1], on average losing around $12 \%$ of total body weight in the first 6 months and up to $45 \%$ over 3 years [2]. Physical activity (PA) and exercise reduce comorbidities related to obesity, such as asthma and sleep problems [3], insulin resistance, hypertension, and hypercholesterolemia [4]. Furthermore, exercise plays an important role in the amount of weight regained following weight loss and helps to reduce weight progressively [5]. Given the increasing number of people with extreme obesity [6], the proven shortand long-term effectiveness of bariatric surgery [7], and the possibilities presented by exercise in relation to the maintenance and improvement of risk factors [8], PA could help those patients who suffer weight regain after bariatric surgery [9].

Previous studies have investigated weight loss caused by exercise following bariatric surgery, generally suggesting the suitability of PA for improving bariatric surgery outcomes [10-12]. However, in many cases, the training program is poorly reported [13]; the exercise type, volume, and intensity are briefly mentioned; and the sample size, selection criteria, and follow-up time vary greatly across studies [14]. The EFIBAR randomized controlled trial (Ejercicio Físico tras cirugía BARiátrica, Physical Exercise after Bariatric Surgery) aims to investigate over 1 year the effects of a 16-week supervised exercise program, initiated immediately after bariatric surgery, on weight loss, body composition, cardiometabolic risk, physical fitness, and quality of life in patients with severe/extreme obesity. Even if additional weight loss is not achieved [14], exercise following bariatric surgery may help to maintain lean body mass, improve cardiovascular health and psychological well-being, and increase adherence to PA, among other benefits [9]. The results of this study may help to assess the effectiveness of exercise as a therapeutic complement to bariatric surgery.

\section{Materials and Methods}

\section{Study Design, Protocol Registration, and Reporting}

The EFIBAR study is a parallel-group, superiority, randomized controlled trial (RCT) registered at www.clinicaltrials. gov (NCT03497546) on April 13, 2018, before the enrollment of participants began (May 1,2018). The design of the study, as well as this protocol manuscript, follows the SPIRIT reporting guideline [15].

\section{Recruitment and Eligibility Criteria}

\section{Recruitment}

The recruitment process takes place through the Bariatric Surgery Units of Torrecárdenas University Hospital and Hospital Mediterráneo, both located in the city of Almería (Spain). In both centers, the surgical team includes surgeons accredited by the Spanish Society for Obesity and Metabolic Surgery (SECO), performing the interventions under the same criteria.

\section{Eligibility Criteria}

The participants' inclusion and exclusion criteria are listed in Table 1.

\section{Sample Size}

Assuming an alpha error of 0.05 and a power of $80 \%$, a total of 66 patients ( $\mathrm{n}=33$ patients per group) are needed to detect an effect (between group difference) of at least 0.7 standard deviations [16] in the \% of total weight loss (\% TWL). Anticipating a potential follow-up loss of up to $20 \%$, a total of 80 patients will be recruited (i.e., 40 per group). Based on previous literature and our surgical team's experience, 60 $80 \%$ of the recruited participants are expected to be women.

\section{Randomization}

Participants are randomly assigned to the experimental (EG) or control group (CG). A member of our research team (statistician), not involved in any other section of the study, created a computer-generated sequence of simple randomization [17]. The sequence is concealed using sealed and opaque envelopes numbered sequentially. Only patients who meet all the selection criteria, sign an informed consent, and finally undergo the surgery receive a randomization number, corresponding to the 
Table 1 Eligibility criteria

Inclusion criteria $\quad$ Exclusion criteria

Age $18-60$ yrs.

BMI $\geq 40 \mathrm{~kg} / \mathrm{m}^{2}$ (or $\geq 35 \mathrm{~kg} / \mathrm{m}^{2}$ with comorbid conditions)

Severe psychiatric or neurological disorders such as schizophrenia, epilepsy, Alzheimer's, Parkinson's, personality disorders, eating behavior disorders, untreated depression, or suicidal tendencies

Acceptable surgical risk (defined by the approval of an anesthetist)

Obesity maintained for over 5 years

Adrenal or thyroid pathology that might cause obesity

Failure of previous treatments

Not presenting contraindications for supervised physical exercise

Uncontrolled addiction to alcohol or drugs

To reside in the city of Almería (or willingness/predisposition to attend the training sessions 3 times a week over 16 weeks)

allocation sequence previously generated. After the surgery, at medical discharge, a nurse opens the envelope in front of the patient, in the absence of any blinded member of the research team.

\section{Blinding}

Because of the type of intervention (exercise program), it is not possible to mask either the patients or the surgeon, given that participants inevitably reveal the group allocation during postoperative follow-up consultations. However, researchers responsible for evaluation and data analysis are blinded to the group assignment (blind evaluator and blind data analyst, respectively). In addition, personal trainers in charge of implementing the exercise program are not part of the assessment team.

\section{Data collection}

\section{Assessment Protocol}

Each participant receives three complete clinical assessments over the study: (1) pre-surgery evaluation, 5-12 days before the surgery; (2) post-intervention evaluation, 5-10 days after completing the intervention phase (i.e., approximately 4

Table 2 Summary of the time-point measurements of the study outcomes

\begin{tabular}{|c|c|c|c|c|c|c|c|}
\hline Measurement & $\begin{array}{l}\text { Pre- } \\
\text { surgery }\end{array}$ & $\begin{array}{l}\text { Month } \\
1\end{array}$ & $\begin{array}{l}\text { Month } \\
3\end{array}$ & $\begin{array}{l}\text { Post-intervention } \\
\text { (month 4) }\end{array}$ & $\begin{array}{l}\text { Month } \\
6\end{array}$ & $\begin{array}{l}\text { Month } \\
9\end{array}$ & $\begin{array}{l}\text { Follow-up (month } \\
12 \text { ) }\end{array}$ \\
\hline \multicolumn{8}{|l|}{ Primary outcome } \\
\hline Weight loss & $\mathrm{x}$ & $\mathrm{x}$ & $\mathrm{x}$ & $\mathrm{x}$ & $\mathrm{x}$ & $\mathrm{x}$ & $\mathrm{x}$ \\
\hline \multicolumn{8}{|l|}{ Secondary outcomes } \\
\hline Body composition $^{\mathrm{a}}$ & $\mathrm{x}$ & & & $\mathrm{x}$ & & & $\mathrm{x}$ \\
\hline CVD risk biomarkers ${ }^{\mathrm{b}}$ & $\mathrm{x}$ & & & $\mathrm{x}$ & & & $\mathrm{x}$ \\
\hline Central and brachial BP, resting HR, PWV & $\mathrm{x}$ & & & $\mathrm{x}$ & & & $\mathrm{x}$ \\
\hline HRV & $\mathrm{x}$ & & & $\mathrm{x}$ & & & $\mathrm{x}$ \\
\hline Health-related fitness and physical activity ${ }^{c}$ & $\mathrm{x}$ & & & $\mathrm{x}$ & & & $\mathrm{x}$ \\
\hline Health-related quality of life (SF-36v2) & $\mathrm{x}$ & & & $\mathrm{x}$ & & & $\mathrm{x}$ \\
\hline Health-related quality of life (EQ-5D) & $\mathrm{x}$ & $\mathrm{x}$ & $\mathrm{x}$ & $\mathrm{x}$ & $\mathrm{x}$ & $\mathrm{x}$ & $\mathrm{x}$ \\
\hline $\begin{array}{l}\text { Symptomatology and function of hip/knee osteoarthritis } \\
\text { (WOMAC) }\end{array}$ & $\mathrm{x}$ & & & $\mathrm{x}$ & & & $\mathrm{x}$ \\
\hline Depression, anxiety, and stress (DASS-21) & $\mathrm{x}$ & & & $\mathrm{x}$ & & & $\mathrm{x}$ \\
\hline $\begin{array}{l}\text { Emotional, psychological, and social well-being } \\
\text { (MHC-SF) }\end{array}$ & $\mathrm{x}$ & & & $\mathrm{x}$ & & & $\mathrm{x}$ \\
\hline
\end{tabular}

$B P$ blood pressure $(\mathrm{mm} / \mathrm{Hg}), C V D$ cardiovascular diseases, $D A S S-21$ Depression, Anxiety and Stress Scale short form, EQ-5D European Quality of Life5 Dimensions, $H R$ heart rate (bpm), $H R V$ heart rate variability, $M H C-S F$ Mental Health Continuum-Short Form, $P W V$ pulse wave velocity (m/seg), $S F$ 36v2 36-item Short-Form Health Survey, WOMAC Western Ontario and McMaster Universities Osteoarthritis Index

${ }^{a}$ Body fat $(\%)$, fat mass $(\mathrm{kg})$, fat-free mass $(\mathrm{kg})$, body mass index (BMI; $\left.\mathrm{kg} / \mathrm{m}^{2}\right)$, waist and hip circumferences $(\mathrm{cm})$

${ }^{\mathrm{b}}$ Total cholesterol (mg/dl), HDL-cholesterol (mg/dl), LDL-cholesterol (mg/dl), triglycerides (mg/dl), glucose (mg/dl), insulin $(\mu \mathrm{UI} / \mathrm{ml}), \mathrm{glycated}$ hemoglobin HbA1c (\%), insulin resistance [homeostasis model assessment, (HOMA-I)], high-sensitivity C-reactive protein [hs-CRP, (mg/dl)], tumor necrosis factor [TNF-alpha $(\mathrm{pg} / \mathrm{ml})]$, interleukin 6 [IL-6, (pg/ml)], alanine aminotransferase (ALT, U/L), aspartate aminotransferase (AST, U/L) and alkaline phosphatase (ALP, U/L)

${ }^{\mathrm{c}}$ Cardiorespiratory fitness $\left(\mathrm{VO}_{2 \mathrm{max}}, \mathrm{METs}\right)$, hand-grip strength (kg), 30-s chair-stand test (number of repetitions), back scratch test (cm), and objectively measured physical activity (accelerometry) 
months after the surgery); and (3) follow-up evaluation, 1 year after the surgery (Figure 1).

\section{Primary Outcome}

The percentage of total weight loss (\%TWL) has been recommended for expressing weight loss after surgery $[18,19]: \%$ $\mathrm{TWL}=[$ (pre-surgery weight - post-surgery weight $) /($ pre-surgery weight) $] \times 100$. In the EFIBAR study, weight is measured using a bioimpedance device (InBody 270, Biospace Co., USA).

\section{Secondary Outcomes}

1. Body composition. Electric bioimpedance (InBody 270, Biospace Co., USA; Lookin'Body 120 software) is used to measure fat mass (FM, $\mathrm{kg}$ ), fat-free mass (FFM, $\mathrm{kg}$ ), and percentage of body fat (\%BF). All participants are asked to urinate before the assessment, and to fast for at least $2 \mathrm{~h}$. Height is measured using a portable system (SECA 213, Hamburg, Germany) with the patient shoeless in a standing position. Body mass index (BMI) is calculated as weight $(\mathrm{kg})$ divided by height squared $\left(\mathrm{m}^{2}\right)$. Waist and hip circumferences are measured in $\mathrm{cm}$ with an anthropometric tape, following standardized guidelines [20].

2. Cardiovascular diseases risk biomarkers. Fasting blood samples are collected and processed by the Sistema Sanitario Público de Andalucía (SSPA) Biobank, according to standard procedures: (2.1) lipid profile: total cholesterol (mg/dl), HDL-cholesterol (mg/dl), LDLcholesterol (mg/dl), and triglycerides ( $\mathrm{mg} / \mathrm{dl}) ;(2.2)$ glycemic control: glucose $(\mathrm{mg} / \mathrm{dl})$, insulin $(\mu \mathrm{UI} / \mathrm{ml})$, glycated hemoglobin HbAlc (\%), and insulin resistance (homeostasis model assessment, (HOMA-I), [21]); (2.3) chronic inflammation: high-sensitivity C-reactive protein [hs$\mathrm{CRP},(\mathrm{mg} / \mathrm{dl})]$, tumor necrosis factor alpha [TNF-alpha $(\mathrm{pg} / \mathrm{ml})]$ and interleukin 6 [IL-6, (pg/ml)]; (2.4) liver metabolism enzymes: alanine aminotransferase (ALT, U/L), aspartate aminotransferase (AST, U/L), and alkaline phosphatase (ALP, U/L).

3. Blood pressure and arterial stiffness: brachial and central blood pressures $(\mathrm{mm} / \mathrm{Hg})$, resting heart rate $(\mathrm{HR}, \mathrm{bpm})$ and arterial stiffness [pulse wave velocity, PWV $(\mathrm{m} / \mathrm{seg})]$ are measured using a Mobil-O-Graph ${ }^{\circledR}$ oscillometry-based pulse analysis system (IEM GmbH, Stolberg, Germany) [22, 23]. Three readings are recorded with the participant resting quietly in a sitting position for $10 \mathrm{~min}$, the cuff placed on the upper left arm around the brachial artery, and the palm facing upwards.

4. Heart-rate variability $(H R V)$. Measurements take place in the morning between 8:30 AM and 11:30 $\mathrm{AM}$ in a quiet, temperature-controlled room (22$24^{\circ} \mathrm{C}$ ). Participants are requested not to exercise or to drink caffeinated and/or alcoholic beverages for $24 \mathrm{~h}$ prior to the examination and to fast for at least $3 \mathrm{~h}$. Participants are instructed to breathe normally and not to talk or fidget while measurements are being taken. Duration of RR intervals is recorded using a Polar V800 telemetry heart-rate monitor (Polar Electro Oy, Kempele, Finland). The electrode belt is dampened and placed just below the chest muscles. Heart rhythm is recorded over $10 \mathrm{~min}$ at a sampling frequency of $1000 \mathrm{~Hz}$ with the patient seated. Kubios Premium software (v.3.2, Heart Rate Analysis, University of Eastern Finland) is used to process the raw HRV data [24, 25].

5. Health-related fitness (HRF) and physical activity (PA). (5.1) Cardiorespiratory fitness is assessed using a

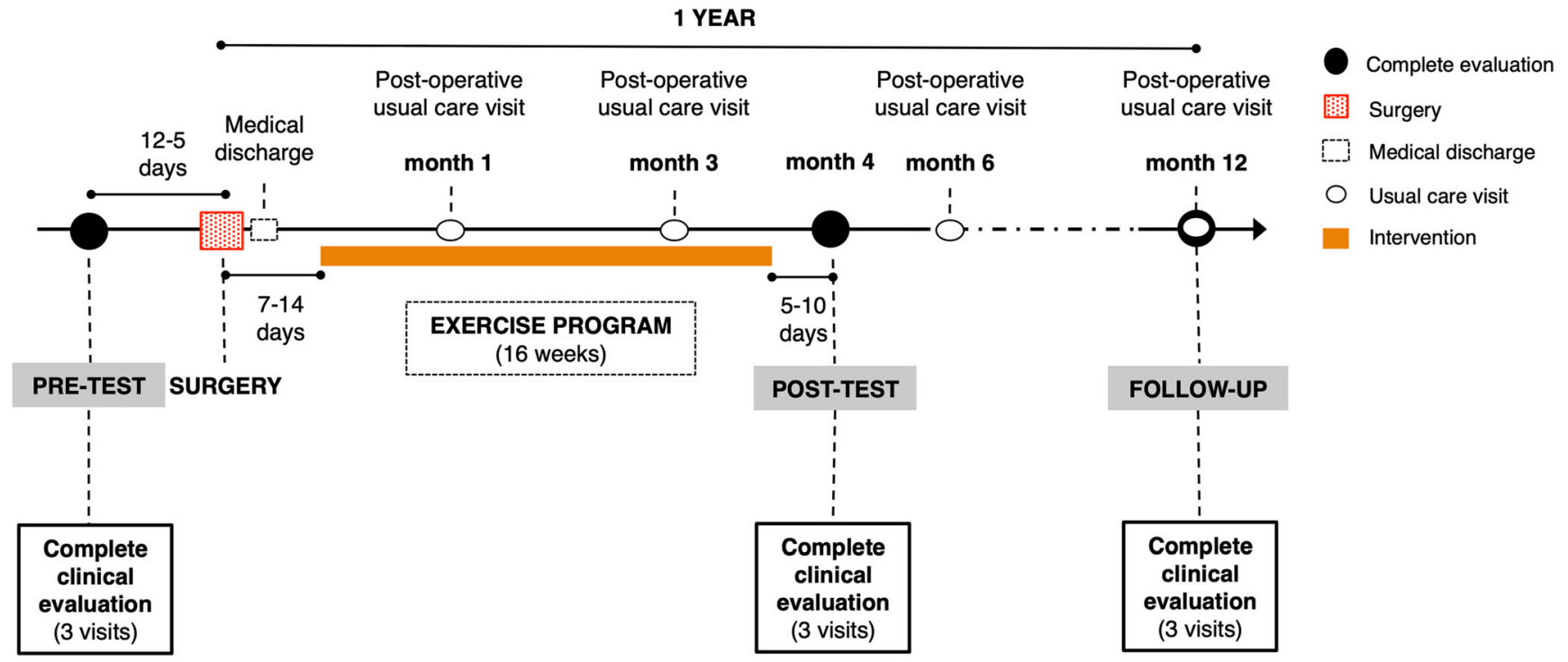

Figure 1 Data collection diagram. 
maximal treadmill test following the Bruce protocol [26, 27]. Maximal oxygen uptake $\left(\mathrm{VO}_{2 \max }\right)$ in METs is estimated based on time to exhaustion. Rate of perceived exertion (RPE, Borg ${ }_{1-10}$ scale), maximum HR (bpm), and recovery $\operatorname{HR}(1,2$, and 3 min afterwards, bpm) are also recorded. (5.2) Hand-grip strength $(\mathrm{kg})$ is measured using a digital hand dynamometer (T.K.K. 5401 Grip-D; Takei, Tokyo, Japan). The patient stands with the shoulder slightly abducted $\left(\sim 10^{\circ}\right)$, the elbow extended, and the forearm and wrist in a neutral position. Each participant performs the test three times (alternately with both hands) allowing a 1-min rest between measurements. Grip span is adapted to hand size differently for men and women [28]. (5.3) Lower body muscular strength: 30 -s chair stand test. This measures the number of full stand-ups from a seated position that participants can complete in $30 \mathrm{~s}$ with arms folded across chest [29]. (5.4) Upper body (shoulder) flexibility: back scratch test $(\mathrm{cm})$. With one hand reaching over the shoulder and one up the middle of the back, the number of centimeters between the extended middle fingers is recorded (plus or minus). The best of two trials is used for analysis [29]. (5.5) Objectively measured PA and sedentary time are assessed by accelerometry (ActiGraph; ActiLife version 6.11 .7 software) [30, 31]. Patients wear a triaxial accelerometer on the hip for $24 \mathrm{~h}$ (except for bathing or water activities) that records acceleration in all three movement axes (in min/day) of PA performed over a total of 7 days. The information is collected over a 60 -s period at a sampling rate of $30 \mathrm{~Hz}$ [30]. The criterion for considering a record valid is to fulfill a minimum of $10 \mathrm{~h}$ a day for 4 days [30].

6. Patient-reported outcomes. (6.1) Health-related quality of life is assessed using the Spanish version of the 36-item Short-Form Health Survey (SF-36v2) [32], together with the European Quality of Life-5 Dimensions (EQ-5D) [33]. (6.2) Symptomatology and function of hip/knee osteoarthritis is measured using the WOMAC (Western Ontario and McMaster Universities) Osteoarthritis Index $[34,35]$. (6.3) Depression, anxiety and stress are assessed using the Depression, Anxiety and Stress Scale short form (DASS-21) [36]. (6.4) Emotional, psychological, and social well-being are measured using the Mental Health Continuum-Short Form (MHC-SF) [37].

7. Cost-effectiveness analysis (CEA) and cost-utility analysis (CUA). The costs of the two treatments under consideration (bariatric surgery and bariatric surgery plus exercise) will be investigated from a dual perspective: the National Health Service and the patient. The first approach will consider the surgery, exercise program, prescription medication, sick leave, postoperative complications, and readmission rates. The patient-centered approach will additionally include the time and effort requiring each treatment, as well as any possible dietary and informal care costs. Effectiveness will be evaluated in terms of changes observed in the main clinical outcomes, i.e., weight loss and cardiometabolic risk. On the other hand, utility will be evaluated as variation in quality of life experienced by participants (quality-adjusted life years, QALY), using SF-36 and EQ-5D questionnaires. All primary and secondary outcomes are shown in Table 2.

\section{Control Variables and Other Parameters to Be Recorded}

Different variables are recorded that may influence the study results even though they are not part of the intervention: (1) sex, age, educational level, marital status, occupational status, and income level; (2) personal history of obesity (obesity duration), cardiovascular disease, hypertension, obstructive sleep apnea (OSA), type 2 diabetes, and medication use; (3) surgical technique (see below); (4.1) adherence to Mediterranean diet [38, 39]; and (4.2) usual intake estimates of food groups, energy, and nutrients using a selfadministered semi-quantitative food-frequency questionnaire (FFQ) [40] and 24-h recalls.

\section{Intervention}

\section{Bariatric Surgery}

Three different techniques are used in this study, all of them carried out using laparoscopic surgery: sleeve gastrectomy (SG), gastric bypass (GB), and one anastomosis gastric bypass (OAGB). The technique used for each participant is decided by a multidisciplinary team of endocrinologists, nutritionists, psychologists, and surgeons, based on patient's BMI, comorbidities, and age, among other considerations. The distribution of the three procedures ( $\mathrm{SG}, \mathrm{GB}$, and OAGB) is expected to be the same in the two groups (control vs. experimental).

\section{Control Group (CG, Usual Care)}

Participants in both groups receive the postoperative followup routinely prescribed in both medical centers, according to international standards [41]. During the first 4 weeks, all patients maintain a semi-liquid diet. Medical consultations are scheduled at months $1,3,6,9$, and 12 after the surgery, with special attention to nutritional status. Written counseling is given, focused on the benefits of a healthy diet and regular physical activity.

\section{Experimental Group (EG, Usual Care + Supervised Exercise)}

The EFIBAR training program has been published elsewhere [42] and was designed and reported following the CERT 
guidelines [43]. The program begins 7-14 days after surgery, exercising 3 times a week over 16 weeks (first 4 weeks of familiarization), using 60-min sessions that includes (1) warm up [light aerobic activity at 50-65\% of HR reserve (HRR)], (2) compensatory training (i.e., core stability and stabilizer muscle exercises), (3) strength training (whole body exercises progressing from 1 to 3 sets, from 12 to 6 repetitions per set, from 24 to 10 repetitions maximum (RM) $(\approx 50$ to $75 \%$ of $1 \mathrm{RM}$ ), (4) aerobic training (on a treadmill, progressing from 15 to $25 \mathrm{~min}$, from 65 to $85 \%$ of HRR), and (5) cool down (static and dynamic stretching exercises) [42] (Figure 2). Maximum HR is recorded during the maximum treadmill test, while resting HR is taken from the HRV assessment, both included in the pre-surgery evaluations. Resting HR is reassessed twice throughout the program (sessions 12th and 36th), and the corresponding aerobic training zones are properly re-calculated. Two members of the research team, with BSc degrees in Sport Sciences and at least 2 years of experience as personal trainers, are in charge of implementing the exercise program. This is performed individually and supervised, with a 1:1 ratio unless participants report schedule unavailability (allowing, in this case, two patients with the same personal trainer) [42]. Attendance, punctuality, training HR, rate of perceived exertion (RPE and ONMI), mood, adverse events, and extra physical activity, among others, are recorded daily. Performing at least $80 \%$ of all planned training sessions will be considered a successful attendance rate. WhatsApp messages and videos are sent to participants every Friday and every month, respectively, to encourage adherence throughout the intervention [42].

\section{Statistical Analysis}

To test whether the randomization has worked effectively, baseline values will be compared for potential clinically important differences. The difference between groups in the change (post-pre) in \% TWL (primary outcome) and all secondary outcomes will be analyzed using the generalized linear model, with the post-pre difference as the dependent variable, and the group (EG or CG), time (16 weeks or 12 months), and their interaction, as the fixed effects. The effect size $(95 \%$ confidence interval) and statistical significance will be reported for the effects of group (between-subjects), time (intra-subject), and the group $\times$ time interaction. The primary analysis will follow per-protocol analysis (only including participants with available data who attend at least $80 \%$ of the exercise sessions). Sensitivity analyses will be performed using the intention-to-treat (ITT) principle. For that purpose, any missing data will be replaced through multiple imputations using regression models with age, type of surgical technique, and baseline values as predictors. The analyses will be adjusted for baseline values plus relevant confounding variables. Stata v.13.1 or higher (StataCorp LP, College Station, TX) will be used to conduct the analyses, and the statistical significance will be set at $\mathrm{P}<.05$.

\section{Discussion}

Obesity is certainly one of the most serious and prevalent health problems currently challenging our society. In less than 5 years (by 2025), obesity is expected to reach a global prevalence of $20 \%$, with almost half of that corresponding to severe/extreme obesity [44]. The burden that this group of patients poses to the health and economic systems is remarkable [45], as a direct result of the treatment costs (usually longterm) but also because of the interruption of their contribution to the production system. Any strategy that can improve their prognosis and allow them to return to society with recovered functional capacity will have a huge impact, not just on them but also on their communities, leading to increased quality of life, productivity, and economic growth.

Our study will primarily investigate whether weight loss 1 year after surgery is greater among those who followed the exercise program. More interestingly, we will be able to test whether this exercise program can mitigate the decrease in muscle mass that normally accompanies bariatric surgery [46]. In addition, this study includes multiple health-related outcomes other than weight loss and body composition, such as lipid and glycemic profiles, chronic inflammation, liver biomarkers, physical fitness, PA, and health-related quality of life. Our experience in previous PA intervention studies [47-52] leads us to believe that the aforementioned health outcomes are more sensitive to exercise and can even be improved in the absence of weight loss [53, 54]. A significant improvement in any of these parameters with no additional weight loss would be a plausible - and very interesting finding.

\section{Conclusion}

The EFIBAR study will provide new insights into the multidimensional benefits of exercise in adults with severe/extreme obesity following bariatric surgery. Using a wide variety of clinical, psychological, and socioeconomic indicators, this study will compare the combination of supervised exercise and usual care with usual care alone. We hypothesize that a 16-week exercise program using strength and aerobic training, implemented immediately after bariatric surgery, will improve weight loss, body composition, cardiometabolic risk, fitness levels, PA, health-related quality of life, and psychological well-being, to a greater extent than the usual care. By using cost-effectiveness and cost-utility analyses, we will also test whether the designed intervention is not only 


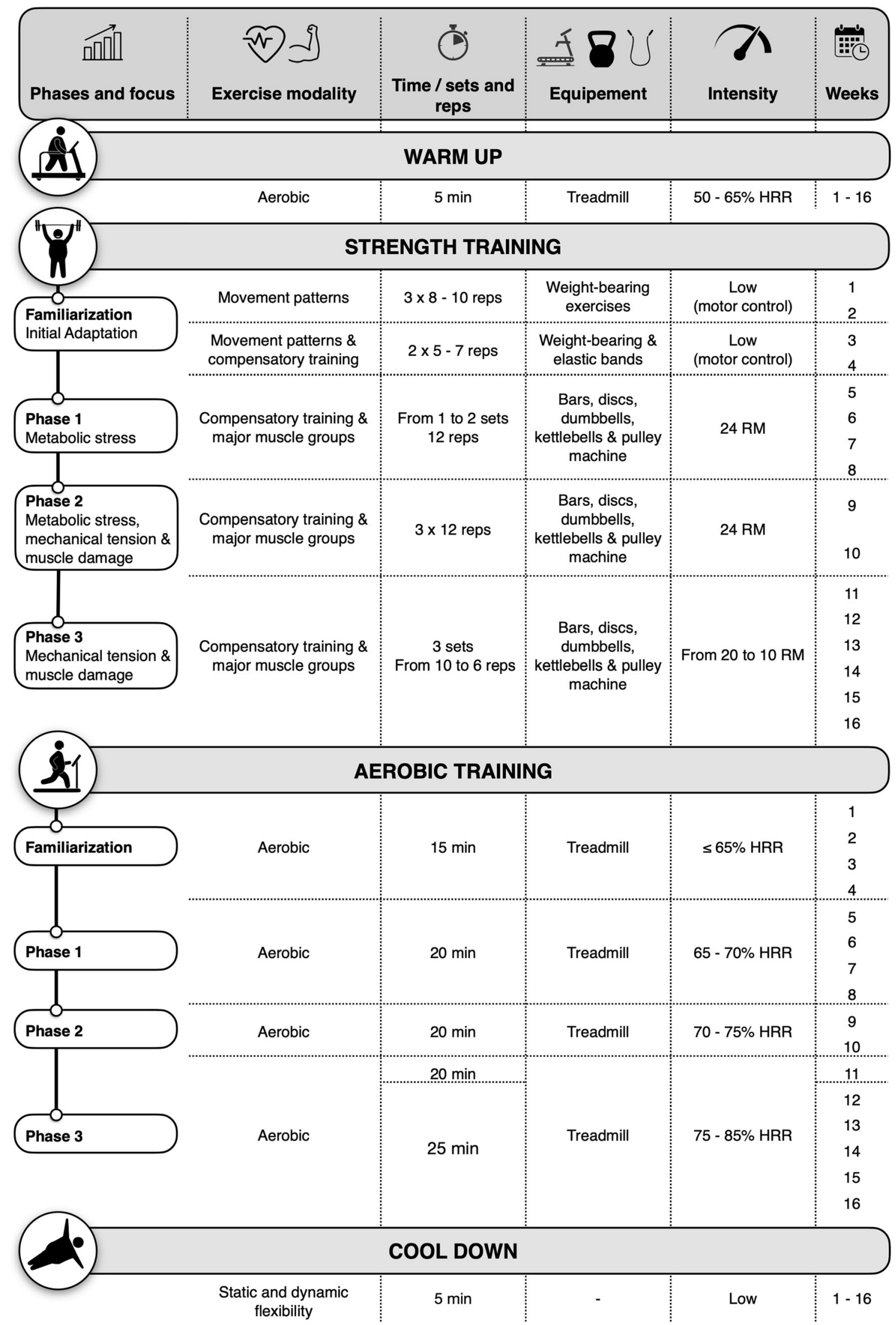

Figure 2. Overview of the EFIBAR training program (for more details, see [42]). HRR: heart-rate reserve, min: minutes, reps: repetitions, RM: repetition maximum. 
feasible and safe, but also effective and useful from an economic standpoint.

Acknowledgements The authors would like to thank all participants for their generous involvement in the study. We would also like to acknowledge the staff and technicians from both hospitals (Torrecárdenas University Hospital and Hospital Mediterráneo), from the SSPA Biobank ( $\mathrm{M}^{\mathrm{a}}$ Eugenia Carrillo Gil, $\mathrm{M}^{\mathrm{a}}$ Mar Berenguel Ibáñez), from the Andalusian Center for Sports Medicine ((CAMD), Adela Cis Spoturno, $\mathrm{M}^{\mathrm{a}}$ Carmen Ibáñez Gil), as well as Francisco Escribano Sotos from the UCLM, and Pablo Molina García from the UGR.

Funding Open Access funding provided thanks to the CRUE-CSIC agreement with Springer Nature. This work was supported by the Spanish Ministry of Economy and Competitiveness (MINECO), Plan Nacional de I+D+i calls RETOS 2016 (reference DEP2016-74926-R) and RETOS 2018 (reference RTI2018-093302-A-I00). EM-R and MAI were funded by the Spanish Ministry of Science, Innovation and Universities (FPU18/01107 and FPU17/01158, respectively). AH-M and LL-S were funded by the pre-doctoral fellowship program of the University of Almería. The funding sources approved this study although they had no role in the design and will not have any role during its execution, analyses and interpretation of the data, writing manuscripts, or decision to submit for publication.

\section{Declarations}

Ethics approval and consent to participate This study protocol was approved by the Ethics Committee of the Torrecárdenas University Hospital (Almería, Spain) prior to the start of the recruitment process (case $\mathrm{N}^{\circ}$ 76/2016). For each new participant being considered, the surgical team provides a comprehensive description of the project, including the general purpose, the assessment protocols, and the intervention program. Informed consent is obtained from all participants included in the study. All procedures are in accordance with the 1964 Helsinki declaration and its later amendments.

Conflict of interest The authors declare that they have no conflict of interest.

Open Access This article is licensed under a Creative Commons Attribution 4.0 International License, which permits use, sharing, adaptation, distribution and reproduction in any medium or format, as long as you give appropriate credit to the original author(s) and the source, provide a link to the Creative Commons licence, and indicate if changes were made. The images or other third party material in this article are included in the article's Creative Commons licence, unless indicated otherwise in a credit line to the material. If material is not included in the article's Creative Commons licence and your intended use is not permitted by statutory regulation or exceeds the permitted use, you will need to obtain permission directly from the copyright holder. To view a copy of this licence, visit http://creativecommons.org/licenses/by/4.0/.

\section{References}

1. Buchwald $\mathrm{H}$, Avidor $\mathrm{Y}$, Braunwald $\mathrm{E}$, et al. Bariatric surgery: a systematic review and meta-analysis. J Am Med Assoc. 2004;292: 1724-37.

2. Wolfe BM, Kvach E, Eckel RH. Treatment of obesity: weight loss and bariatric surgery. Circ Res. 2016;118:1844-55.

3. Freitas PD, Silva AG, Ferreira PG, et al. Exercise improves physical activity and comorbidities in obese adults with asthma. Med Sci Sports Exerc. 2018;50:1367-76.
4. Swift DL, Johannsen NM, Lavie CJ, et al. The role of exercise and physical activity in weight loss and maintenance. Prog Cardiovasc Dis. 2014;56:441-7.

5. Swift DL, Johannsen NM, Lavie CJ, et al. The role of exercise and physical activity in weight loss and maintenance. Prog Cardiovasc Dis. 2014;56:441-7.

6. Basterra-Gortari FJ, Beunza JJ, Bes-Rastrollo M, et al. Increasing trend in the prevalence of morbid obesity in Spain: from 1.8 to 6.1 per thousand in 14 years. Rev Esp Cardiol. 2011;64:424-6.

7. Ricci C, Gaeta M, Rausa E, et al. Long-term effects of bariatric surgery on type II diabetes, hypertension and hyperlipidemia: a meta-analysis and meta-regression study with 5-year follow-up. Obes Surg. 2015;25:397-405.

8. Lakka TA, Bouchard C. Physical activity, obesity and cardiovascular diseases. Handb Exp Pharmacol. 2005:137-63.

9. Coen PM, Carnero EA, Goodpaster BH. Exercise and bariatric surgery: an effective therapeutic strategy. Exerc Sport Sci Rev. 2018:46:262-70.

10. Livhits M, Mercado C, Yermilov I, et al. Exercise following bariatric surgery: systematic review. Obes Surg. 2010;20:657-65.

11. Egberts K, Brown WA, Brennan L, et al. Does exercise improve weight loss after bariatric surgery? A systematic review. Obes Surg. 2012;22:335-41.

12. Bellicha A, Ciangura C, Poitou C, et al. Effectiveness of exercise training after bariatric surgery - a systematic literature review and meta-analysis. Obes Rev. 2018;19:1544-56.

13. Soriano-Maldonado A, Villa-González E, Ferrer-Márquez M, et al. Replicability of exercise programs following bariatric surgery. Atherosclerosis. 2018;278:330-1.

14. Carretero-Ruiz A, Olvera-Porcel M, del Carmen Cavero-Redondo I, et al. Effects of exercise training on weight loss in patients who have undergone bariatric surgery: a systematic review and metaanalysis of controlled trials. Obes Surg. 2019;29:3371-84.

15. Chan AW, Tetzlaff JM, Altman DG, et al. SPIRIT 2013 statement: defining standard protocol items for clinical trials. Ann Intern Med. 2013;158:200-7.

16. Armitage P, Colton T, (Eds). Encyclopedia of Biostatistics. 2nd ed. Wiley; 2005.

17. Schulz KF, Grimes DA. Generation of allocation sequences in randomised trials: chance, not choice. Lancet. 2002;359:515-9.

18. Van De Laar A. Bariatric outcomes longitudinal database (BOLD) suggests excess weight loss and excess BMI loss to be inappropriate outcome measures, demonstrating better alternatives. Obes Surg. 2012;22:1843-7.

19. Sczepaniak JP, Owens ML, Shukla H, et al. Comparability of weight loss reporting after gastric bypass and sleeve gastrectomy using BOLD Data 2008-2011. Obes Surg. 2015;25:788-95.

20. Norton KI, Eston R. Kinanthropometry and Exercise Physiology. 4th ed. London: Taylor \& Francis Group; 2019.

21. Matthews DR, Hosker JP, Rudenski AS, et al. Homeostasis model assessment: insulin resistance and $\beta$-cell function from fasting plasma glucose and insulin concentrations in man. Diabetologia. 1985;28:412-9.

22. Weiss W, Gohlisch C, Harsch-Gladisch C, et al. Oscillometric estimation of central blood pressure: validation of the Mobil-O-Graph in comparison with the SphygmoCor device. Blood Press Monit. 2012;17:128-31.

23. Papaioannou TG, Argyris A, Protogerou AD, et al. Non-invasive 24 hour ambulatory monitoring of aortic wave reflection and arterial stiffness by a novel oscillometric device: the first feasibility and reproducibility study. Int J Cardiol. 2013;169:57-61.

24. Ibacache P, Cárcamo P, Miranda C, et al. Improvements in heart rate variability in women with obesity: short-term effects of sleeve gastrectomy. Obes Surg. 2020;30:4038-45.

25. Martínez-Rosales E, Sola-Rodríguez S, Vargas-Hitos JA, et al. Heart rate variability in women with systemic lupus erythematosus: 
association with health-related parameters and effects of aerobic exercise. Int J Environ Res Public Health. 2020;17:9501.

26. Bruce RA, Blackmon JR, Jones JW, et al. Exercising testing in adult normal subjects and cardiac patients. Pediatrics. 1963;32: $742-56$.

27. Guazzi M, Adams V, Conraads V, et al. Clinical recommendations for cardiopulmonary exercise testing data assessment in specific patient populations. Circulation. 2012;126:2261-74.

28. Ruiz-Ruiz J, Mesa JLM, Gutiérrez A, et al. Hand size influences optimal grip span in women but not in men. J Hand Surg [Am]. 2002;27:897-901.

29. Rikli RE, Jones CJ. Development and validation of a functional fitness test for community- residing older adults. J Aging Phys Act. 1999;7:129-61.

30. Sasaki JE, John D, Freedson PS. Validation and comparison of ActiGraph activity monitors. J Sci Med Sport. 2011;14:411-6.

31. Donaldson SC, Montoye AHK, Tuttle MS, et al. Variability of objectively measured sedentary behavior. Med Sci Sports Exerc. 2016;48:755-61.

32. Alonso J, Prieto L, Anto JM. The Spanish version of the SF-36 Health Survey (the SF-36 health questionnaire): an instrument for measuring clinical results. Med Clin (Barc). 1995;104:771-6.

33. Badia X, Roset M, Montserrat S, et al. The Spanish version of EuroQol: a description and its applications. European Quality of Life scale. Med Clin (Barc). 1999;112:79-85.

34. McConnell S, Kolopack P, Davis AM. The Western Ontario and McMaster Universities Osteoarthritis Index (WOMAC): a review of its utility and measurement properties. Arthritis Rheum. 2001;45: 453-61.

35. Escobar A, Quintana JM, Bilbao A, et al. Validation of the Spanish version of the WOMAC questionnaire for patients with hip or knee osteoarthritis. Clin Rheumatol. 2002;21:466-71.

36. Bados A, Solanas A, Andrés R. Psychometric properties of the Spanish version of Depression, Anxiety and Stress scales (DASS). Psicothema. 2005; 17:679-83.

37. Echeverría G, Torres M, Pedrals N, et al. Validation of a Spanish Version of the Mental Health Continuum-Short Form Questionnaire. Psicothema. 2017;29:96-102.

38. Schröder H, Fitó M, Estruch R, et al. A Short screener is valid for assessing Mediterranean diet adherence among older Spanish men and women. J Nutr. 2011;141:1140-5.

39. Martínez-González MA, García-Arellano A, Toledo E, et al. A 14item Mediterranean diet assessment tool and obesity indexes among high-risk subjects: the PREDIMED trial. PLoS One. 2012;7: e43134.

40. Fernández-Ballart JD, Piñol JL, Zazpe I, et al. Relative validity of a semi-quantitative food-frequency questionnaire in an elderly Mediterranean population of Spain. Br J Nutr. 2010;103:1808-16.

41. Mechanick JI, Youdim A, Jones DB, et al. Clinical practice guidelines for the perioperative nutritional, metabolic, and nonsurgical support of the bariatric surgery patient-2013 update: cosponsored by American Association of Clinical Endocrinologists, the Obesity Society, and American Society fo. Obesity. 2013;21:S1-27.
42. Villa-González E, Barranco-Ruiz Y, Rodríguez-Pérez MA, et al. Supervised exercise following bariatric surgery in morbid obese adults: CERT-based exercise study protocol of the EFIBAR randomised controlled trial. BMC Surg. 2019;19:127.

43. Slade SC, Dionne CE, Underwood M, et al. Consensus on Exercise Reporting Template (CERT): explanation and elaboration statement. Br J Sports Med. 2016;50:1428-37.

44. Di Cesare M, Bentham J, Stevens GA, et al. Trends in adult bodymass index in 200 countries from 1975 to 2014: a pooled analysis of 1698 population-based measurement studies with 19.2 million participants. Lancet. 2016;387:1377-96.

45. Anekwe CV, Jarrell AR, Townsend MJ, et al. Socioeconomics of Obesity. Curr Obes Rep. 2020;9:272-9.

46. Nuijten MAH, Monpellier VM, Eijsvogels TMH, et al. Rate and determinants of excessive fat-free mass loss after bariatric surgery. Obes Surg. 2020;30:3119-26.

47. Labayen I, Medrano M, Arenaza L, et al. Effects of exercise in addition to a family-based lifestyle intervention program on hepatic fat in children with overweight. Diabetes Care. 2020;43:306-13.

48. Cadenas-Sánchez C, Mora-González J, Migueles JH, et al. An exercise-based randomized controlled trial on brain, cognition, physical health and mental health in overweight/obese children (ActiveBrains project): Rationale, design and methods. Contemp Clin Trials. 2016;47:315-24.

49. Sanchez-Delgado G, Martinez-Tellez B, Olza J, et al. Activating brown adipose tissue through exercise (ACTIBATE) in young adults: rationale, design and methodology. Contemp Clin Trials. 2015;45:416-25.

50. Coll-Risco I, Acosta-Manzano P, Borges-Cosic M, et al. Body composition changes following a concurrent exercise intervention in perimenopausal women: the FLAMENCO Project Randomized Controlled Trial. J Clin Med. 2019;8:1678.

51. Carbonell-Baeza A, Aparicio VA, Ortega FB, et al. Does a 3-month multidisciplinary intervention improve pain, body composition and physical fitness in women with fibromyalgia? Br J Sports Med. 2011;45:1189-95.

52. Soriano-Maldonado A, Morillas-de-Laguno P, Sabio J, et al. Effects of 12-week aerobic exercise on arterial stiffness, inflammation, and cardiorespiratory fitness in women with systemic LUPUS erythematosus: non-randomized controlled trial. J Clin Med. 2018;7:477.

53. Coen PM, Menshikova EV, Distefano G, et al. Exercise and weight loss improve muscle mitochondrial respiration, lipid partitioning, and insulin sensitivity after gastric bypass surgery. Diabetes. 2015;64:3737-50.

54. Coen PM, Tanner CJ, Helbling NL, et al. Clinical trial demonstrates exercise following bariatric surgery improves insulin sensitivity. J Clin Invest. 2015;125:248-57.

Publisher's Note Springer Nature remains neutral with regard to jurisdictional claims in published maps and institutional affiliations. 